\title{
MÉNINGITE VERMINEUSE
}

\section{Par J. HAUTEFEUILLE}

Les réactions méningées de l'helminthiase ne sont pas très fréquentes. Beaucoup de médecins restent sceptiques sur leur réalité. Il arrive cependant que des enfants, condamnés par des confrères, guérissent grâce à un vermifuge, administré par une guérisseuse soignant par les simples.

Il s'agit parfois seulement de méningisme et on a longtemps parlé de pseudo-méningite vermineuse. Mais on peut observer aussi une véritable méningite avec fièvre et modification cytologique et chimique du liquide céphalo-rachidien, dont le tableau simule, à s'y méprendre, la méningite tuberculeuse. Le diagnostic est souvent fait tardivement, au moment de l'expulsion, spontanée ou due à un purgatif, d'helminthes dont on ne soupçonnait pas la présence, expulsion qui est suivie de la disparition de tous les phénomènes méningés.

C'est un cas de ce genre que j'ai observé.

Le 16 juin 1931, un jeune homme de 19 ans se réveille à 4 heures du matin avec la tête lourde et vomit plusieurs fois.

Je le vois à 7 heures. La céphalée est devenue violente, la température est de $38^{\circ} 9$, le pouls à 100 . Je constate une légère raideur de la nuque, pas de signe de Kernig, ni de photophobie, le ventre est souple, non douloureux.

Le 17 et le 18, la céphalée est de plus en plus forte, l'intolérance gasIrique absolue, la langue est saburrale ; il existe de la constipation.

Le 19, le malade entre à l'hôpital. On constate une légère ébauche de Kernig, de la rétention d'urine.

Le 20, pour éliminer un état typhique avec réaction méningée, nous faisons un séro-diagnostic qui est négatif.

Une ponction lombaire indique :

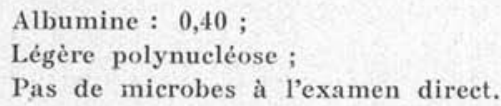

Le 21, devant ce résultat, nous injectons $20 \mathrm{~cm}^{3}$ de sérum antiméningococcique intra-rachidien et $40 \mathrm{~cm}^{3}$ sous la peau.

Le 22, même état, même traitement.

Annales de Parasitologie, $\mathrm{T}$. XIII, N 1 - - Janvier 1935, p. 21-27. 
Le 23 nous recevons le résultat d'un deuxième examen du liquide céphalo-rachidien :

Albumine : 0,50 ;

Lymphocytes : 100 par champ ;

Pas de bacilles de Koch.

On cesse le sérum.

Le 24, la céphalée qui s'était un peu atténuée après les ponctions

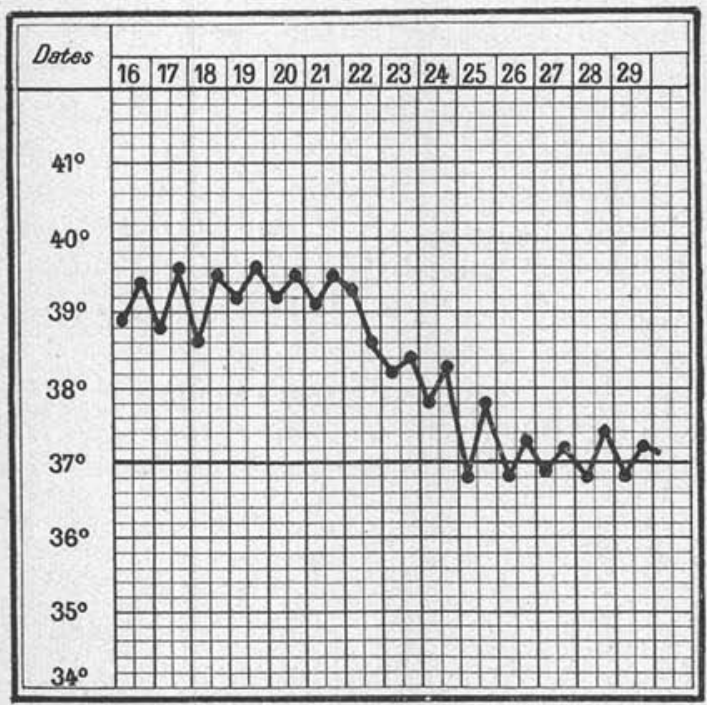

Fig. - Courbe de température du malade atteint de méningite vermineuse

reprend de plus belle; les vomissements continuent. Le malade ne supporte aucune boisson, ni eau de Seltz ni glace.

Le Kernig est devenu net, les réflexes sont très diminués ; il n'existe pas d'hypéresthésie cutanée.

Le malade maigrit rapidement, le ventre est rétracté en bateau.

On l'hydrate par un goutte-à-goutte rectal au sérum glycosé.

Pour éliminer une méningite spécifique, nous faisons pratiquer un Wassermann qui est négatif.

La température s'est abaissée peu à peu. Le 25 elle n'atteint que $37^{\circ} 8$ puis ne dépasse plus $37^{\circ} 2$.

Les symptômes persistent, bien qu'atténués le 26 et le 27 . Le 28, le malade peut absorber un peu de bouillon.

Le 29 il rejette dans les selles un Ascaris lumbricoides. A partir de ce 
moment, la céphalée et les vomissements cessent, le malade peut s'alimenter assez rapidement.

Il se lève le 2 juillet et quitte l'hôpital le 4 . Il ne ressent plus aucun malaise, mais il est très affaibli ; il a maigri de $9 \mathrm{~kg}$. pendant sa maladie et ne reprend son travail que le 21 juillet, après trois semaines de convalescence à la campagne.

En 1932, il fait un an de service militaire sans aucune indisponibilité puis reprend son service de domestique de maison. Il n'a plus ressenti aucun trouble.

En résumé, ce malade a présenté un syndrome méningitique net, à début brusque, avec céphalée, vomissements, constipation, fièvre qui a duré 12 jours.

La polynucléose constatée au premier examen du liquide céphalorachidien, la violence des symptòmes nous ont fait croire d'abord à une méningite à méningocoques, puis la lymphocytose du second examen à une méningite tuberculeuse.

Ce dernier diagnostic ne paraît pas pouvoir être maintenu, bien que des rémissions très longues soient possibles. Mais le sujet est un garçon robuste, sans aucun antécédent et qui n'a plus jamais présenté aucun malaise.

On pourrait penser à une méningite à lymphocytes curable, j'en ai autrefois présenté plusieurs cas à la Société médicale de Picardie, et ce sujet a été traité dans la thèse du $\mathrm{D}^{\mathrm{r}}$ Jacquemard de St-Valéry.

Un fait est bien net, c'est la disparition de tous les symptômes au moment de l'évacuation d'un Ascaris; il est difficile d'admettre une simple coïncidence.

Nous pensons donc qu'il s'agit réellement d'une méningite vermineuse. Cette méningite constitue donc une nouvelle catégorie de ces méningites lymphocytaires curables.

Cette maladie a été décrite pour la première fois en 1835 par Lebon, de Besançon. Elle fut longtemps qualifiée de méningisme ou de pseudo-méningite.

La ponction lombaire a pu, dans des cas frustes, donner un liquide céphalo-rachidien en tous points normal.

Mais en 1922, Guillain et Gardin ont trouvé, au cours d'une méningite vermineuse certaine, une réaction nette du liquide : 0,56 d'albumine, 276 leucocytes.

Depuis, les faits analogues se sont multipliés : Beutter : 18 lymphocytes ; Aurand : 1 gr. 60 d'albumine, 165 éléments ; Lafforgue : lymphocytose très abondante ; Gautier : 400 éléments ; Abel et Brenas : 250 éléments.

Lorsque la formule leucocytaire a été pratiquée, il s'est agi le plus 
souvent d'une formule mixte avec lymphocytose prédominante (70 p. 100).

Exceptionnellement, le cas d'Abel et Brenas signalait de la polynucléose ( 65 p. 100$)$.

Dans notre cas, une première phase de polynucléose a été suivie de lymphocytose.

L'éosinophilie a été recherchée par ces derniers auteurs. Elle manquait dans le liquide céphalo-rachidien; mais l'examen a été pratiqué le $13^{\circ}$ jour, au début de la convalescence.

L'éosinophilie sanguine était de 1 p. 100. Chez le malade de Beutter l'éosinophilie sanguine était de 3 p. 100 à la même période.

Nous n'avons pas trouvé de document sur l'éosinophilie à la période d'état de la méningite. Elle serait peut-être au début un bon élément de diagnostic.

On peut, en tous cas, conclure de l'existence de ces réactions du liquide céphalo-rachidien à la réalité d'une méningite vraie dûe aux helminthes.

Le tableau clinique est très semblable à celui d'une méningite tuberculeuse avec un amaigrissement rapide. Cependant quelques anomalies peuvent attirer l'attention.

La violence et l'irrégularité des symptômes nerveux contrastent avec la régularité du pouls et des mouvements respiratoires. L'évolution par crises subites avec des rémissions profondes, une amélioration marquée passagère après les ponctions lombaires, l'absence de signes oculaires, la disparition des symptômes après l'expulsion des vers, telles sont les particularités signalées par les auteurs.

Mon malade a aussi été très soulagé momentanément après chaque ponction lombaire. J'ai été surtout frappé chez lui par la violence des vomissements, par l'intolérance absolue de l'estomac, plus accentuée que dans la plupart des méningites tuberculeuses.

Le diagnostic est difficile par la clinique. La lymphocytose rachidienne habituelle n'élucide pas le problème. Peut-être l'évaluation de l'éosinophilie donnerait-elle une indication intéressante ; elle n'a pas été faite chez notre malade.

La recherche des œufs dans les selles est facile. Si elle est négative, elle élimine l'origine vermineuse de la méningite ; si elle est positive, il peut s'agir évidemment de la coïncidence possible de parasitisme intestinal et d'une méningite dûe à une autre cause, mais il est indiqué d'administrer un vermifuge qui servira de traitement d'épreuve.

La pathogénie de la méningite vermineuse est discutée.

On admettait autrefois la théorie réflexe par irritations des ter- 
minaisons nerveuses de l'intestin par les parasites. Cette théorie explique peut-être certains troubles fugaces causés par les vers : réactions vaso-motrices, toux spasmodique, méningisme, convulsions, mais elle ne parait pas pouvoir s'appliquer aux méningites caractérisées avec réaction cytologique.

La theorie toxique parait plus vraisemblable. La toxicité des vers, de l'Ascaris en particulier, est démontrée. La manipulation des Ascaris provoque des picotements et du gonflement des doigts, du prurit, de la conjonctivite si les mains sont portées aux yeux, de l'éternuement, des phénomènes généraux fébriles. L'injection d'extrait d'Ascaris détermine chez les animaux des symptômes d'intoxication.

Pour Guiart, les toxines vermineuses sont de plusieurs sortes, les unes ont un pouvoir hémolytique et produisent l'anémie ; d'autres, qui auraient plutôt le caractère de ferments, affectent plus particulièrement les centres nerveux ; d'autres, à caractère alcaloïdique, ont une action paralysante sur les muscles. Ces toxines semblent donc pouvoir provoquer une méningite.

D'autres exemples de méningite toxique sont connus. La méningite saturnine s'accompagne de réaction lymphocytaire du liquide céphalo-rachidien et parfois rappelle à s'y méprendre la méningite tuberculeuse.

C'est à l'intoxication que Guillain et Gardin attribuent la méningite qu'ils ont observée; les toxines vermineuses, parvenant dans le liquide céphalo-ráchidien par la voie des plexus choroïdes ou par toute autre voie sanguine, détermineraient des modifications circulatoires dans la séreuse arachnoïdo-pie-mérienne et provoqueraient le passage de l'albumine et l'exode leucocytaire dans le liquide céphalo-rachidien.

Une troisième théorie attribue les accidents de l'helminthiase à un choc anaphylactique analogue à celui qui se produit au moment de la ponction des kystes hydatiques.

Weinberg et Julien, en instillant sur la conjonctive des chevaux un liquide périentérique d'Ascaris, ont vu certains d'entre eux s'affaisser, être agités de secousses convulsives et mourir en quelques minutes. Ils ont attribué ce phénomène à un choc anaphylactique chez des animaux sensibilisés par une infestation ascaridienne antérieure.

Simonin, de Nancy et Morenas, de Lyon, ont réalisé des accidents anaphylactiques nets chez les cobayes à l'aide d'extraits d'Ascaris.

La réaction de Bordet, réalisée avec le sérum des sujets porteurs 
de vers intestinaux, l'éosinophilie sanguine et l'émission de cristaux de Charcot-Leyden dans les selles sont des indices biologiques de sensibilisation.

Cette théorie s'applique plutôt aux manifestations cutanées, prurit nasal, urticaire, œdèmes, à la toux, à l'asthme vermineux, aux convulsions et au simple méningisme.

Dans la méningite vraie, il paraît difficile d'admettre le rôle exclusif de l'anaphylaxie. Il faut faire la part principale à l'intoxication qui sensibilise le système nerveux et provoque la réaction méningée. Mais du choc anaphylactique peuvent dépendre les crises qui entrecoupent l'évolution de la méningite.

On pourrait rapprocher ces faits des méningites sériques causées par ufn traitement aux sérums thérapeutiques.

Quoi qu'il en soit de ces théories, une notion pratique est à retenir, c'est l'existence de méningites d'origine vermineuse.

Dans tous les cas où l'origine tuberculeuse ou autre de la méningite ne sera pas certaine, il sera prudent de faire une recherche des œufs dans les selles, d'évaluer l'éosinophilie ou de faire, à défaut de ces examens, un traitement d'épreuve.

On obtiendra parfois une guérison miraculeuse et on évitera des erreurs de pronostics fâcheuses pour la réputation du praticien.

\section{RÉSUMÉ}

Un jeune homme de 19 ans est atteint de céphalée avec vomissements et intolérance gastrique absolue. La température reste à $39^{\circ}$ pendant 6 jours, puis s'abaisse graduellement à $37^{\circ}$ le dixième jour.

Les symptômes subissent une rémission après chaque ponction lombaire et ne disparaissent que le quatorzième jour, après rejet d'un Ascaris lumbricoìdes dans les selles.

Une première ponction lombaire indique de la polynucléose, une seconde de la lymphocytose. L'auteur conclut, du fait de la réaction leucocytaire du liquide céphalo-rachidien, à l'existence d'une véritable méningite vermineuse et termine par des considérations sur la pathogénie de cette maladie.

\section{BibliographiE}

Abet et Brenas. - Méningite grave d'origine ascaridienne. Archines de médecine des enfants, juillet $1925, \mathrm{n}^{\circ} 7$.

Aurand, - Méningite vermineuse. Conconrs médical, 1922, p. 1930. 
Gauthier (de Genève). - Un cas de méningite vermineuse. Soc. méd. des hópitaux de Paris, 16 mars 1923, p. 429.

Guiart. - Biologie et rôle pathogène des parasites intestinaux. Nouveau traité de pathologie générale de Bouchard.

Guillain et Gardin. - Contribution à l'étude des méningites de l'helminthiase. Bull. Acad. de Méd., 1922, p. 308.

Hautefeumle. - Les méningites lymphocytaires curables. Gazette médicale de Picardie, mars 1926 , p. 117.

Jacquemard. - Les méningites lymphocytaires curables. Thèse de Paris, 1926.

LAFFongev. - Forme typho-méningitique de la lombricose avec réaction méningée lymphocytique. Soc. méd. de Toulouse, janvier 1923.

Morenas. - Anaphylaxie dans l'helminthiase intestinale. Archives de l'appareil digestif, nov. 1926 , p. 1.035 .

Simonin. - Introduction à l'étude des toxines vermineuses. Thèse de Nancy, 1919-1920.

Turcan. - Les symptômes méningés de l'ascaridiose. Presse méd., sept. :922, p. 844 .

Weinberg et Julien. - Accidents mortels observés chez le cheval à la suite d'instillation de toxines ascaridiennes. C.R. Soc. Biol., 1913, p. 1162.

Ecole de médecine dAmiens. 